\title{
Cellule de coordination des flux sortants des réanimations en période de Covid-19
}

\section{Coordination Unit of Outgoing Flow from Intensive Care Unit during COVID-19 Crisis}

\author{
M. Langlois - M. Borel $\cdot$ O. Clovet $\cdot$ V. Justice $\cdot$ C. Spuccia $\cdot$ M. Raux \\ Reçu le 29 juin 2020 ; accepté le 20 juillet 2020 \\ (C) SFMU et Lavoisier SAS 2020
}

Résumé La pandémie de Covid-19 a inscrit l'hôpital au cœur d'une crise sanitaire de cinétique longue. Le système de santé a dû dans un premier temps accepter cette notion de crise déstructurante et piloter dans l'incertitude. Un des enjeux majeurs était d'éviter la saturation du système, notamment l'accessibilité à la réanimation. À la demande de la cellule de crise du groupe hospitalier AP-HPSorbonne Université, l'équipe Dynamo a dû apporter des propositions permettant de libérer des places en réanimation. C'était la stratégie retenue pour éviter une mise en tension de l'hôpital. La cellule Dynamo, avec l'accord du directeur médical de crise, a ouvert un flux entre les réanimations expertes et des unités créées de novo (publiques et privées). Cette équipe est le fruit d'une préparation conjointe entre le département médico-universitaire DREAM et le service médical du RAID. Elle a permis d'organiser et d'effectuer dans de bonnes conditions sanitaires et sécuritaires le transfert d'une centaine de patients entre les réanimations d'îlede-France. L'objectif était une répartition cohérente pour

M. Langlois $(\square) \cdot$ M. Borel $\cdot$ O. Clovet $\cdot$ M. Raux

Département d'anesthésie-réanimation, hôpital Pitié-Salpêtrière, Assistance publique-Hôpitaux de Paris (APHP),

groupe hospitalier universitaire AP-HP-Sorbonne Université, F-75013 Paris, France

e-mail : matthieulanglois@me.com

M. Langlois

Service médical du RAID (Recherche, Assistance, Intervention, Dissuasion), force d'intervention de la Police nationale, domaine du Bel-Air, route de Gisy, F-91570 Bièvres, France

V. Justice $\cdot$ C. Spuccia

Coordination des prélèvements d'organes et de tissus, hôpital Pitié-Salpêtrière, APHP,

groupe hospitalier universitaire AP-HP-Sorbonne Université, F-75013 Paris, France

\section{Raux}

Inserm, UMRS 1158, neurophysiologie respiratoire expérimentale et clinique, Sorbonne Université,

F-75013 Paris, France maintenir une capacité d'accueil dans les réanimations les plus spécialisées et impactées par l'intensité des soins. Pour cela, la cellule Dynamo a défini des critères médicaux de patients éligibles au transfert. La méthodologie utilisait quatre boucles indépendantes : le service demandeur, l'équipe de transfert, le vecteur de transfert et le service receveur. Cette organisation a offert agilité et autonomie. Nous publions ce retour d'expérience pour partager les bases méthodologiques et humaines de notre organisation afin d'inspirer d'autres cellules innovantes en cas de situations sanitaires exceptionnelles.

Mots clés Covid-19 · Gestion de crise · Transfert interhospitalier · Innovation

Abstract The COVID-19 pandemic has put the hospital infrastructure into the difficulty of a long time public health crisis. The health care system had to accept the concept of destructuring crisis and ultimately piloting with uncertainty. The key factor during the crisis was to avoid the saturation of the care system especially for the intensive care unit. Upon the hospital medical crisis unit request, the DYNAMO team was accountable to propose solutions for "outflow". Under the crisis medical director's green light, the DYNAMO unit has opened flow between the hospital intensive care unit and step down units created de novo (public and private). DYNAMO is the outcome of the collaboration and joint preparation between the university medical department DREAM and RAID Tactical medical unit allowing the use of technical tools and adding the tactical spirit into the hospital frame. This collaboration has supported the transfer in secure conditions of about 100 patients across the intensive care units with a consistent distribution of patients in order to maintain the most efficient intensive care units impacted by the crisis able to accept an influx of new patients. To achieve this, the DYNAMO team defined medical criteria to determine a patient's eligibility to be transferred under the team transfer supervision. The methodology is formed by 4 independent 
loops: the requester service, the transfer team, the medium for transfer and the receiver service. This model appeared to be simple, agile and autonomous. We are delighted to share our lessons learned on the methodology and human organization with the emergency care community.

Keywords COVID-19 · Crisis management · Inter-hospital transfer $\cdot$ Innovation

\section{Introduction}

Dans toute crise sanitaire, l'hôpital doit se mettre en ordre de marche pour être pleinement opérationnel. Sur une crise à cinétique longue comme une pandémie, il est important de bâtir une stratégie qui permet aux structures hospitalières de ne pas arriver à saturation [1,2]. Ce pilotage est vertical et horizontal. Les cellules de crise fixent le raisonnement tactique [3]. Il est indispensable pour cela de constituer des unités détachées du pilotage et chargées de proposer des solutions innovantes en fonction des données évolutives de la crise. L'objectif est de permettre au décideur de voir audelà des plans - pas forcément adaptés à la crise en cours pour ne pas tomber dans un effet tunnel incapacitant. À la demande du directeur médical de crise (DMC) du groupe hospitalier Assistance publique-Sorbonne Université (AP-HP-SU), une cellule appelée " Dynamo » a été créée afin d'organiser et de coordonner le transfert de plus de 100 patients des réanimations de AP-HP-SU au plus fort de la crise afin de soulager les structures, sans devoir solliciter les Samu, eux-mêmes devant faire face à un nombre d'appels en augmentation considérable. Nous souhaitons, à travers ce retour d'expérience, témoigner des outils utilisés et les partager avec l'ensemble de la communauté hospitalière et préhospitalière.

\section{Justification d'un besoin}

La crise est, par définition, une situation brutale qui entraîne une rupture [4]. On parle de hors cadre quand la structure, les procédures et l'humain sont immédiatement projetés dans un nouveau concept non prédéfini. Les référentiels techniques et humains disponibles ne peuvent plus apporter de solution éclairante. Ce temps est chaotique, et l'analyse de la crise impose de l'humilité dans un premier temps puis la capacité à faire naître de la cohésion, de la confiance et de l'innovation [5]. La désorganisation spatiale et temporelle touche l'ensemble des organisations. La pandémie correspond parfaitement à cette définition [6]. Le système de santé a dû dans un premier temps accepter cette notion de crise déstructurante, analyser sans pouvoir tout comprendre puis piloter dans l'incertitude [7].
En pareille circonstance, les structures hospitalières se dotent de cellule de crise. Les attentats de 2015 avaient illustré parfaitement l'importance d'une culture de crise au sein de l'hôpital [8]. En 2019, le Guide de préparation et à la gestion des tensions hospitalières et des situations sanitaires exceptionnelles inscrit le DMC comme un élément majeur de la cellule de crise [9]. Son rôle est d'apporter une aide technique au pilotage médical de l'hôpital soumis à une forte tension. À côté du directeur d'établissement, il apporte une culture médicale et de pilotage de crise. Il est le coordinateur médical de l'ensemble des partenaires médicaux hospitaliers comme extrahospitaliers [10].

Dans le cas de la pandémie de Covid-19, une des demandes de la cellule de crise de l'AP-HP-SU était de libérer des lits de réanimation en créant du flux entre les réanimations, afin de maintenir une capacité d'accueil permanente pour les patients réanimatoires [11]. Partant du principe que toutes les réanimations n'ont pas le même niveau d'expertise (une salle de surveillance postinterventionelle qui se transforme en quelques heures en unité de réanimation n'a pas la performance d'une réanimation polyvalente experte en ventilation du syndrome de détresse respiratoire aiguë), la cellule Dynamo a proposé que les patients hospitalisés dans des réanimations spécialisées, et dont l'état clinique ne nécessitait pas pareil niveau d'expertise (du fait de leur amélioration par exemple), soient transférés vers des réanimations moins spécialisées. L'hypothèse formulée est que cela créerait du flux entre les réanimations de l'AP-HP-SU en s'appuyant sur une structuration de l'offre des soins de réanimation régionale en quatre niveaux d'unité de réanimation experte, existants avant la crise de Covid-19, aptes à prendre des patients multidéfaillants aux unités de réanimations créées de novo. Cette architecture a engendré des flux optimisés pour contrôler la mise en tension hospitalière [12].

\section{Construction d'une réponse agile au service du directeur médical de crise}

La cellule Dynamo est le fruit d'une préparation conjointe entre le département médico-universitaire DREAM et le service médical du RAID. Le partage entre les médecins de ces deux structures depuis 2015 a permis d'utiliser non seulement des outils techniques, mais aussi l'état d'esprit de la médecine tactique au sein même de l'hôpital [13]. Les formations [14], les exercices, la simulation [15] et les travaux scientifiques menés depuis cinq ans ont validé une capacité médicale à innover dans l'incertitude et sous contraintes fortes. La médecine tactique se résume souvent à «penser différemment » [13] dont s'est inspiré Dynamo pour organiser le transfert de malades de Covid-19.

Les réanimations de l'AP-HP-SU (des hôpitaux PitiéSalpêtrière, Tenon, Saint Antoine, Trousseau où une 
réanimation adulte avait été créée dans un site pédiatrique) avaient la charge de rédiger une demande de transfert selon un formulaire préparé en amont (limitation et synthèse obligatoires des transmissions : pas plus de deux lignes) et l'envoyer à la cellule Dynamo via une adresse boîte e-mail dédiée. La cellule de coordination était par ailleurs dotée d'une ligne téléphonique spécifique et deux ordinateurs connectés aux systèmes informatiques de l'AP-HP. Les critères a priori d'acceptabilité pour un transfert étaient un patient intubé depuis au moins 48 heures, pas de décubitus ventral depuis au moins 24 heures, une $\mathrm{FiO}_{2}$ inférieure à $60 \%$, un support hémodynamique par noradrénaline inférieur à $1 \mathrm{mg} / \mathrm{h}$. La cellule Dynamo appelait chaque jour l'ensemble des structures susceptibles d'accueillir un transfert, suivant le listing de l'Agence régionale de santé (ARS) Île-de-France, couplé au listing de Ramsay Générale de Santé, l'ensemble des structures éligibles à un transfert chaque jour. Ce listing était annoté au quotidien en fonction du retour des équipes éligibles (typologie de défaillance acceptée, perspective de place, distance et manque de matériel). Une fois une place trouvée pour un patient, la cellule Dynamo constituait une équipe de transfert en la composant avec une ambulance non médicalisée (le plus souvent privée), un réanimateur (médecin réanimateur ou anesthésiste-réanimateur) de l'équipe demandeuse ou issue d'une liste de volontaires (anesthésistes-réanimateurs [senior et interne]) de la PitiéSalpêtrière et réanimateurs et infirmiers pédiatres de l'hôpital Trousseau, et d'un aide-réanimateur (chirurgien, infirmier coordinateur, jeune interne). Le matériel de transfert était fourni par l'équipe demandeuse. Un médecin de l'équipe Dynamo était toujours accessible par téléphone pour résoudre toute difficulté médicale ou non, liée au transfert avant, pendant ou après celui-ci. Il était convenu avec l'équipe de transfert qui évaluait le patient dans sa réanimation d'origine qu'en cas de modification de la typologie du patient (dégradation notamment), elle appelle l'équipe Dynamo pour prise de décision quant au transfert. L'équipe de transfert partait avec un kit médicamenteux d'urgence pour sécuriser le transfert (préparé par l'équipe de départ, selon les consignes). En plus des transferts gérés de bout en bout par la cellule Dynamo, elle a recherché des patients conformes aux propositions de transferts interrégionaux organisés par le DMC de l'AP-HP en lien avec le Samu zonal (Samu 75) et l'ARS Île-de-France, afin d'utiliser au mieux ces possibilités exceptionnelles.

L'équipe Dynamo s'est construite dans l'urgence. Elle était composée de trois médecins anesthésistesréanimateurs et de deux infirmières coordinatrices de prélèvement d'organes et de tissu. Chaque membre de la cellule était très complémentaire. Chacun a immédiatement trouvé sa place et son rôle [16]. Il n'existait pas de hiérarchie, ce qui a offert beaucoup de souplesse, de confiance et d'efficacité dans la difficulté. Les médecins anesthésistes-réanimateurs possédaient tous une expérience du préhospitalier et de l'hospitalier. Cela a fortement aidé à la compréhension du problème et à trouver des solutions correspondantes tant pour les exigences de réanimation que pour les conditions de transport des malades. Si c'était une évidence au sein de l'équipe, il était indispensable de fédérer au-delà de la cellule pour mettre en place des organisations complexes et hors cadre. Le management de la cellule Dynamo s'est fait uniquement sur la confiance [17]. Il fallait tout autant donner confiance aux structures hospitalières publiques et privées qu'aux directeurs d'établissements et DMC, aux médecins et chirurgiens assurant les transferts ou encore aux équipes d'ambulanciers qui n'avaient jamais imaginé transférer des patients intubés et ventilés [12]. En donnant du sens à la mission de chacun, l'ensemble de la chaîne Dynamo a permis cette innovation et d'atteindre un objectif partagé [18].

Dans la crise, la situation chaotique doit pousser à retrouver des procédures simples. Le concept du KISS (Keep it simple as stupid) [le plus simple possible, idiot est utilisé dans le sens de " ne complique pas les choses »] dans le management est fort précieux lors d'une mise en tension brutale de la structure et des équipes soignantes [19]. Notre canevas fait de boucles courtes et modulables de type " vecteur par ambulance privée gros volume » apporte souplesse et efficacité.

\section{Enjeu : chronotopie médicale}

La chronotopie est une notion littéraire qui lie dans la narration le temps et l'espace. Le pilotage de crise correspond exactement à ce concept. Les solutions émergentes du pilotage de la crise doivent apporter des solutions qui maîtriseront dans le même temps les contraintes spatiales et temporelles. La doctrine de médecine tactique du RAID pour répondre à un afflux de victimes en cas de tuerie de masse a été modélisée dans cet objectif [8]. La cellule Dynamo s'est inscrite dans ce concept avec une organisation spatiale comprenant les quatre niveaux de réanimations, le choix des vecteurs de transfert et les équipes pour optimiser la gestion de l'espace et du temps. Ce lien opérationnel entre le temps et l'espace évitait une saturation de la disponibilité des lits.

La médecine tactique travaille sur l'optimisation des flux d'évacuation des blessés dans une zone rouge lors de tuerie de masse [20]. Tout est concentré sur cet objectif : limiter le temps entre la blessure et l'évacuation au trauma center. Le matériel, le circuit, le triage et la coordination ont été pensés pour accélérer les flux. Ce concept de flux est travaillé par des équipes hospitalières lors d'exercice de simulation [15]. La cellule Dynamo est dans cette logique d'optimisation des flux ; à chaque demande, elle trouvait la solution la plus simple et la plus rapide possible pour créer un flux et ainsi libérer un lit de réanimation. Le DMC fixant les objectifs plusieurs fois par jour. 


\section{Résultats de la cellule Dynamo}

L'activité de la cellule Dynamo a commencé le 27 mars 2020 pour s'arrêter le 22 avril 2020. Elle a tenu une permanence quotidienne aux heures ouvrables y compris le week-end. La cellule a reçu 149 demandes, dont 105 ont été effectuées. L'ensemble des demandes et de leurs caractéristiques ont été tracées de façon prospective. L'âge médian des patients proposés au transfert était de 62 ans [interquartile [IQR] : 51-67]. Le nombre médian de jours de ventilation avant d'être proposé pour un transfert était de sept jours [IQR : 4-13]. Le pic du nombre de demandes de transferts a eu lieu le $1^{\text {er }}$ avril avec 19 transferts effectués pour 23 demandes (Fig. 1).

La réanimation qui a été le plus à l'origine de demandes était la réanimation médicale de Tenon $(n=28)$, puis la réanimation médicale neurologique de la Pitié-Salpêtrière $(n=$ 20 ) et enfin la réanimation chirurgicale de Saint-Antoine ( $n$ =19) (Fig. 2). La destination principale des patients a été la réanimation temporaire éphémère créée au sein du bâtiment de chirurgie ambulatoire de la Pitié-Salpêtrière $(n=40)$, puis la réanimation adulte créée au sein de l'hôpital pédiatrique Trousseau $(n=7)$ et la clinique Geoffroy-Saint-Hilaire $(n=$ 6) (Fig. 3). Quatorze patients sont partis en dehors de la région Île-de-France, pris en charge par TGV, avion ou hélicoptère par les moyens du Samu zonal ou du service de santé des armées. La modalité de transfert la plus fréquemment utilisée dans le cadre de Dynamo était l'association d'une ambulance privée avec une équipe de deux ambulanciers, un médecin anesthésiste-réanimateur, un chirurgien. L'ambulance en question était dans ce cadre-là le plus souvent équipé par le matériel de la réanimation de départ.

Les demandes non honorées ont été pour l'essentiel dues à une dégradation avant transfert $(n=22)$, à la difficulté pour trouver une place pour un patient avec nécessité persistante d'hémodialyse $(n=7)$ ou en surpoids majeur $(n=4)$. Aucun incident majeur ne s'est déroulé au cours des transferts coordonnés par Dynamo.

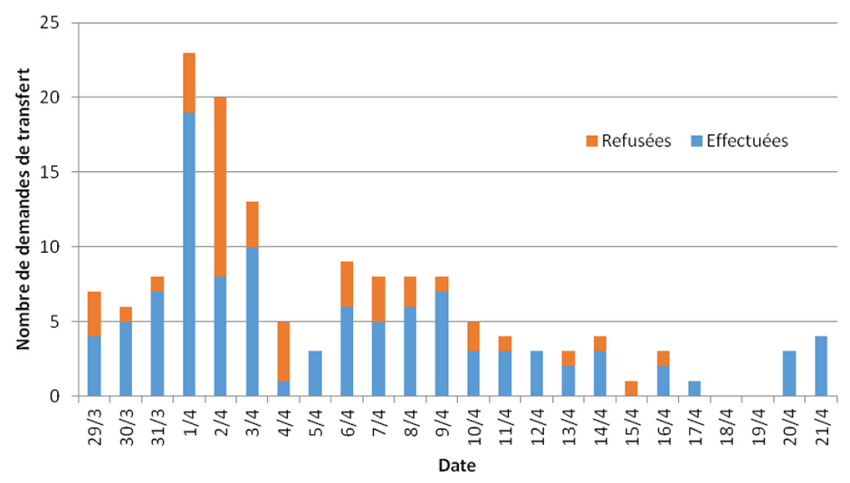

Fig. 1 Nombre de demandes traitées au cours du temps
L'organisation du transfert d'un patient résultait de l'association de quatre boucles indépendantes et maîtrisées :

- le service demandeur : sa connaissance des possibilités offertes par Dynamo, les caractéristiques du patient :

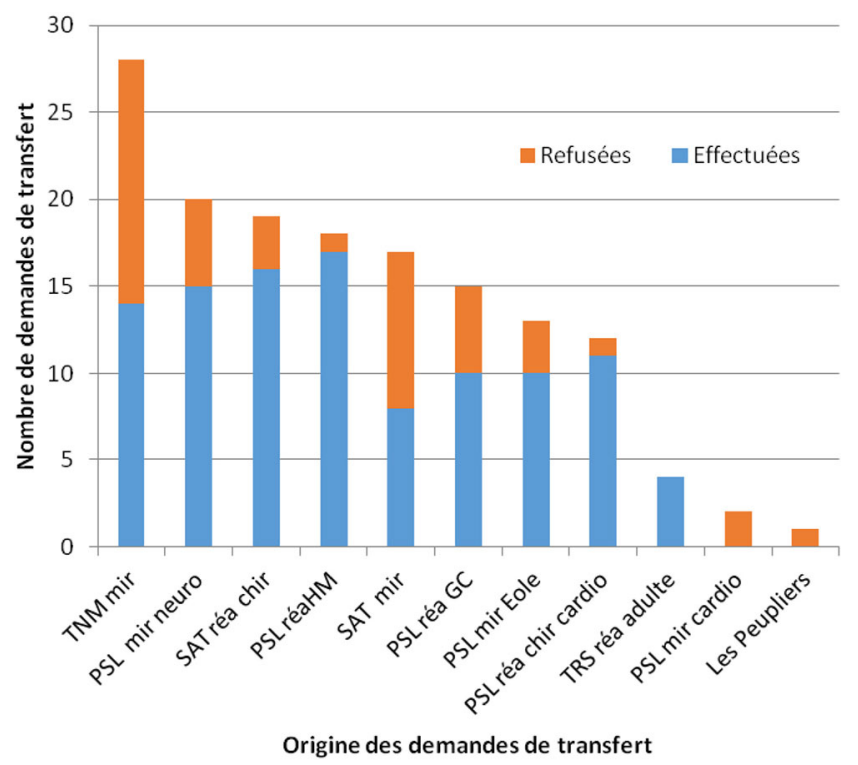

Fig. 2 Origine des demandes et décisions prises (effectuées/refusées). TNN : hôpital Tenon ; SAT : hôpital Saint-Antoine ; PSL : hôpital Pitié-Salpêtrière ; TRS : hôpital Trousseau ; mir : médecine intensive et réanimation; neuro : neurologie ; réa chir : réanimation chirurgicale ; cardio : cardiologie

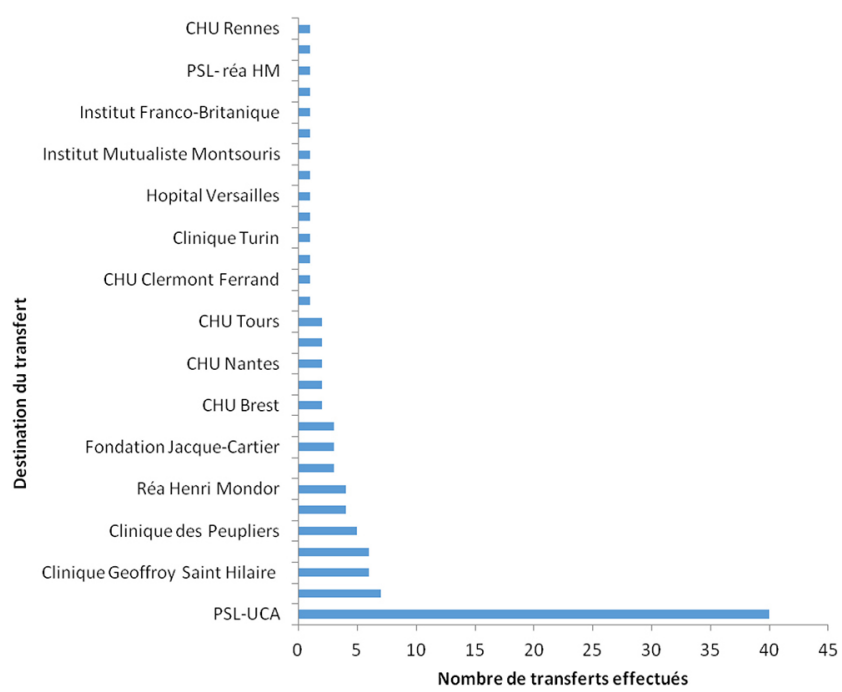

Fig. 3 Destination des patients. PSL-UCA : réanimation unité de chirurgie ambulatoire du CHU de l'hôpital Pitié-Salpêtrière ; TRS-réa : réanimation adulte de Trousseau, PSL-USC - Covid : unités de soins continus Covid du CHU Pitié-Salpêtrière ; CHU : centre hospitalo-universitaire ; $\mathrm{CH}$ : centre hospitalier ; HP : hôpital privé 
nombre de jours de réanimation, niveau de réanimation respiratoire, nombre de défaillances associées, support par vasoconstricteur ;

- les équipes de transfert : ambulanciers, médecins, aidemédecins ;

- le vecteur de transfert : ambulance, train, aérien ;

- le service receveur réanimation de novo ou réanimation existante antérieurement au Covid.

La tâche de Dynamo peut se résumer à la capacité de combiner et de lier ces boucles avec agilité et réactivité. Il revenait à Dynamo de confirmer à chaque maillon de la chaîne son adéquation avec le suivant. Seule Dynamo avait la maîtrise complète de la stratégie.

\section{Enseignements d'une cellule innovante et agile au profit du pilotage de crise hospitalière}

La cellule Dynamo est née et a fonctionné pour accomplir la mission donnée par le DMC. Normalement, cette mission de transfert incombe aux Samu. Leur refus, justifié par d'autres missions prioritaires, a obligé les décideurs hospitaliers à imaginer une solution en dehors de tout cadre. Cette cellule n'existait dans aucun plan préalable. Mais qui réellement utilise les plans lors d'une mise en tension brutale ? Les plans sont là pour encadrer la préparation mais dans le fort de la crise, peu se réfèrent aux plans car nous sommes, par définition, dans le hors cadre [21].

La crise de Covid-19 apporte de très nombreux enseignements. Le rôle des DMC est renforcé. Ils auront besoin dans les crises futures de faire montre de leadership et d'innovation [22]. La cellule Dynamo dont l'objectif était d'offrir des flux sortants à l'hôpital est un exemple. Mais chaque crise est différente et la prochaine fois la problématique sera probablement autre. Une règle reste toujours valable, l'agilité dans l'incertitude est déterminante.

Pour la cellule de crise, il ne doit y avoir aucune limite à l'initiative et à l'innovation. Elle doit donc compter sur des groupes cohérents qui avancent en confiance dans l'incertitude. Charge au DMC et aux cellules de crise de fixer les objectifs et de laisser s'exprimer des cellules opérationnelles du type de Dynamo. Cela répond au principe de centralisation stratégique/décentralisation opérationnelle d'une gestion de crise. Au plus fort de la crise, la cellule Dynamo avait proposé au DMC et organisé une capacité vectorielle de 50 ambulances gros volume capables d'apporter des solutions de transfert vers la province.

Les auteurs sont persuadés que la cellule Dynamo aurait pu être encore plus efficace. Sa création dans l'urgence et dans une situation dégradée n'a pas permis une réflexion à plus large échelle. Les ressources humaines et matérielles disponibles étaient très limitées, et le contexte n'imposait aucune alternative. La cellule n'a, par exemple, reçu aucune aide du Samu, occupé à assurer sa mission d'aide médicale urgente et les transferts d'hospitalisation conventionnelle vers les réanimations. Pour autant, elle s'est mise à sa disposition pour assurer l'identification des patients éligibles aux transferts longue distance. Une meilleure coordination, une stratégie commune et partagée entre les DMC de l'AP-HP et les Samu auraient permis de réaliser certains transferts les plus lourds dans des conditions sanitaires plus confortables. Cela aurait permis à Dynamo d'augmenter le nombre, la distance et la sécurité des transferts. La cellule Dynamo peut à l'avenir s'inscrire dans un partage mieux coordonné. Il parait, pour cela, indispensable d'offrir un cadre légal à ce type de transport interhospitalier médicalisé par une équipe du service hospitalier émetteur. À l'avenir, la cellule Dynamo offre des solutions exportables facilement lors de SSE, mais cela demande quelques améliorations (préparation et entraînement des équipes au hors cadre, partage des enjeux de flux interhospitaliers entre le DMC et le Samu, amélioration de la répartition des moyens par une coordination commune pré- et intrahospitalier, création d'un cadre légal pour ces transferts).

Les auteurs souhaitent par cette communication permettre une exportation de ce type d'initiative. Il repose pour l'essentiel sur un objectif sanitaire prioritaire et utilise au mieux les ressources humaines et matérielles disponibles en situation dégradée.

\section{Conclusion}

La crise de Covid-19 a vu naître de très nombreuses initiatives. Elles ont toutes apporté des solutions aussi bien organisationnelles, techniques qu'humaines. Cette souplesse est probablement une des leçons de cette crise hospitalière. La cellule Dynamo est un exemple parmi d'autres. Créée dans l'urgence et avec très peu de moyens, elle met en avant que la simplicité des solutions apportées est souvent la clé de l'engagement et de la cohésion dans l'incertitude d'une crise. Le DMC, le directeur hospitalier, la cellule de crise hospitalière doivent pouvoir compter sur des cellules d'anticipation et d'innovation et s'entraîner à travailler dans le hors cadre. Le DMC et le directeur doivent savoir motiver une réflexion audacieuse [23].

Liens d'intérêts : Marie Borel déclare un conflit d'intérêt avec la société Medusims (conseiller pédagogique) et avec la société Emergensim (formateur). Matthieu Langlois avec le laboratoire Teleflex (expert). Le Pr. Mathieu Raux et le Dr. Matthieu Langlois sont responsables d'une formation universitaire à la direction médicale de crise au sein de la Faculté de médecine de Sorbonne Université. 


\section{Éléments remarquables}

- Cellule innovante créée dans l'urgence et « hors cadre »

- Utilisation de ressources très économes sur le plan des ressources humaines, des moyens mis à disposition et financier

- Décloisonnement agile entre le public et le privé

- Adhésion collective aux projets Dynamo par le partage de sens et la confiance
Pistes d'amélioration

- L'interaction avec la cellule de crise (DMC) doit être simplifiée pour plus de réactivité en fonction de l'évolution de la crise

- Amélioration des communications avec les acteurs publics et privés pour être encore plus agiles (réseau de communication et application numérique dédiés)

- La cellule de gestion de flux hospitalier doit trouver sa place en coordination avec le Samu et l'Agence régionale de santé (ARS)

- Une cellule d'aide à la gestion de crise doit favoriser la stratégie partagée entre ARS, Samu et les DMC hospitaliers.

\section{Références}

1. Yang C, Tsai SH, Chien WC, et al (2019) The crowd-out effect of a mass casualty incident. Experience of a dust explosion with multiple burn injuries. Medicine 98:1-5

2. Dulion JB, Langlois M, Raux M (2019) Gestion de crise intrahospitalière face à la menace terroriste. Rev Hospit France 586:45-51

3. Samurcay R, Rogalski J (1993) Cooperative work and decisionmaking in emergency management. Travail Hum 56:53-77

4. Lagadec P (2019) Le Temps de l'invention. Femmes et hommes d'État aux prises avec les crises et ruptures en univers chaotique. Préventique. http://www.patricklagadec.net/fr/pdf/LagadecLeTempsdelInvention.pdf (Dernier accès le 23 juin 2020)

5. Lagadec P (2015) Journal de bord des temps chaotiques. Le continent des imprévus (2015) Éd. Manitoba. Les Belles Lettres, Paris

6. Hick JL, Christian MD, Sprung CL (2010) European Society of Intensive Care Medicine's task force for intensive care unit triage during influenza epidemic or mass disaster. Surge capacity and infrastructure considerations for mass critical care. Recommendations and standard operating procedures for intensive care unit and hospital preparations for an influenza epidemic or mass disaster. Intensive Care Med 36:S11-S20

7. Helsloot I, Boin A, Jacobs B, Comfort L (2012) Mega-crises: Understanding the prospects, nature, characteristics and effects of cataclysmic events. Ed. Charles Thomas Publisher, Springfield

8. Service médical du RAID (2015) Médicalisation de l'extrême avant au cours d'une intervention des forces de l'ordre pour prise d'otages : principes régissant la prise en charge médicale et retour d'expérience du RAID. Ann Fr Med Urgence 5:166-75

9. Ministère des Solidarités et de la Santé (2019) Guide d'aide à la préparation et à la gestion des tensions hospitalières et des situations sanitaires exceptionnelles. https://solidarites-sante.gouv.fr/ IMG/pdf/guide_situation_sanitaire_exceptionnelle.pdf (Dernier accès 23 juin 2020)

10. Sorbonne Université (2019) Le directeur médical de crise. https:// dpc.sorbonne-universite.fr/offre-de-formation/formations-pourles-medecins/le-directeur-medical-de-crise-dmc-module-1-de-latheorie-a-la-pratique (Dernier accès 23 juin 2020)
11. Corcoran S, Niven A, Reese J (2012) Critical care management for major disasters: a practical guide to disaster preparation in the intensive care unit. J Intensive Care Med 27:3-10

12. Joynt G, Taylor B, Margalit G, et al (2010) Coordination and collaboration with interface units. Intensive Care Med 36:S21-31

13. Langlois M, Gomez ML, Kerveillant M, Borel M (2019) Gérer la mise en tension brutale des structures hospitalières. Gestion Hospit 595: 202-6

14. Développement professionnel continu, Médecine Sorbonne Université (2018) Bases de la médecine tactique au service des forces d'intervention. https://dpc.sorbonne-universite.fr/offre-de-formation/formations-pour-les-medecins/ (Dernier accès le 23 juin 2020)

15. Borel M, Damm C, Debien B, et al (2019) S'exercer à l'afflux massif de victimes hospitalières... Comment faire ? Ann Fr Med Urg 9:163-72

16. Langlois M (2019) Prise de décision dans l'incertitude. Expérience des médecins tactiques du RAID. LIREC 58:13-7

17. Ghorbanian A, Bahadori M, Nejati M (2012) The relationship between manager's leadership styles and emergency medical technicians' job satisfaction. Australas Med J 5:1-7

18. Gamble MS, Hanners RB, Lackey C, Beaudin CL (2009) Leadership and hospital preparedness: disaster management and emergency services in paediatrics. J Trauma 677:S79-S83

19. Teo W, Lee M, Lim WS (2017) The relational activation of resilience model: how leadership activates resilience in an organizational crisis. J Contingencies Crisis Manag 25:136-47

20. Lauria MJ, Gallo BA, Brooks S, et al (2017) Psychological skills to improve emergency care providers' performance under stress. Ann Emerg Med 70:884-90

21. Service médical du RAID (Recherche, Assistance, Intervention, Dissuasion) (2016) Retour d'expérience des attentats du 13 novembre 2015. Gestion de l'attaque terroriste du Bataclan par les médecins d'intervention de la Police nationale. Ann Fr Med Urgence 6:3-8

22. Subbe C, Kellet J, Barach P, et al (2017) Crisis checklists for inhospital emergencies: expert consensus, simulation testing and recommendations for a template determined by a multiinstitutional and multi-disciplinary learning collaborative. BMC Health Serv Res 17:334

23. Riou B (2016) 13 novembre 2015 : terrorisme, résilience et espoir. Ann Fr Med Urg 6:1-2 